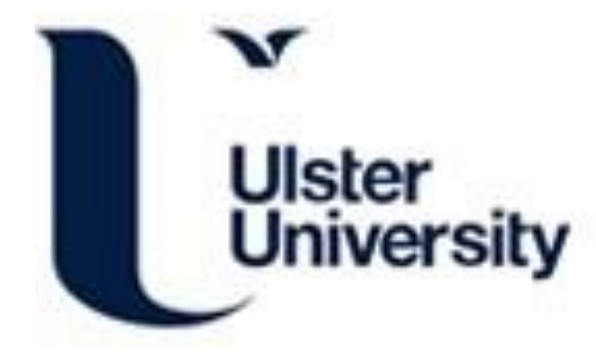

\title{
Developing Mental Health Awareness and Help Seeking in Prison: A Feasibility Study of the State of Mind Sport Programme.
}

Woods, D., Leavey, G., Meek, R., \& Breslin, G. (Accepted/In press). Developing Mental Health Awareness and Help Seeking in Prison: A Feasibility Study of the State of Mind Sport Programme. International Journal of Prisoner Health, 1-30.

Link to publication record in Ulster University Research Portal

\section{Published in:}

International Journal of Prisoner Health

Publication Status:

Accepted/In press: 10/06/2020

\section{Document Version}

Author Accepted version

\section{General rights}

Copyright for the publications made accessible via Ulster University's Research Portal is retained by the author(s) and / or other copyright owners and it is a condition of accessing these publications that users recognise and abide by the legal requirements associated with these rights.

\section{Take down policy}

The Research Portal is Ulster University's institutional repository that provides access to Ulster's research outputs. Every effort has been made to ensure that content in the Research Portal does not infringe any person's rights, or applicable UK laws. If you discover content in the Research Portal that you believe breaches copyright or violates any law, please contact pure-support@ulster.ac.uk. 


\section{International Journal of Prisoner}

Developing Mental Health Awareness and Help Seeking in

Prison: A Feasibility Study of the State of Mind Sport

Programme

\begin{tabular}{|r|l|}
\hline Journal: & International Journal of Prisoner Health \\
\hline Manuscript ID & IJPH-10-2019-0057.R1 \\
\hline Manuscript Type: & Research Paper \\
\hline Keywords: & $\begin{array}{l}\text { Health promoting prison, Mental health, Stigma, Sport Based } \\
\text { Intervention, Feasibility, Mixed methods }\end{array}$ \\
\hline
\end{tabular}

\section{SCHOLARONE ${ }^{\text {M }}$ \\ Manuscripts}




\section{Abstract \\ Purpose}

The high prevalence of mental illness within the prison population necessitates innovative mental health awareness provision. This feasibility study with 75 males (47 intervention; 28 control) evaluated State of Mind Sport, originally developed as a community based mental health and well-being initiative, in a notoriously challenging prison setting.

\section{Design}

A mixed 2 (group) x 2 (time) factorial design was adopted. Questionnaires tested for effects on knowledge of mental health, intentions to seek help, well-being and resilience. For each outcome measure, main and interaction effects $(F)$ were determined by separate mixed factors Analysis of Variance (ANOVA). Two focus groups $(N=15)$ further explored feasibility and were subjected to General Inductive Analysis.

\section{Findings}

A significant Group and Time interaction effect was shown for mental health knowledge, $F(1,72)=4.92, p=0.03, \eta p 2=0.06$, showing a greater post-programme improvement in mental health knowledge score for the intervention group. Focus group analysis revealed an increase in hope, coping efficacy and intentions to engage more openly with other prisoners regarding personal well-being as a result of the SOMS programme. However, fear of stigmatisation by other inmates and a general lack of trust in others remained as barriers to help-seeking.

\section{Originality}

The implications of this study, the first to evaluate a sport-based mental health intervention in prison, are that a short intervention with low costs can increase prisoner knowledge of mental health, intentions to engage in available well-being opportunities and increase a sense of hope, at least in the short term.

- Key words: Feasibility, Mental Health Awareness, Prison Health, Sport Based Intervention, Stigmatisation. 


\section{Introduction}

There is a high prevalence of mental illness within the prison population (WHO, 2014), characterised by estimates of depression, anxiety and stress-related symptoms affecting up to 90\% of prisoners, (Durcan, 2016; Fraser, Gatherer \& Hayton, 2009; Leigh-Hunt \& Perry, 2015). Prisoners with decreased levels of mental health are at further risk of suicide, selfharm, violent assault and victimisation (Fazel, Hayes, Bartellas, Clerici \& Trestman, 2016). Behaviours which can help buffer against the risks identified, such as help seeking or supporting others are often stigmatised and perceived as a weakness within prison culture (Ricciardelli, Maier \& Hannah-Moffat, 2015). Therefore, in order to encourage protective behaviours and reduce the risks of self-harm and suicide, there exists a need to implement mental health awareness activities in prison settings (MacNamara \& Mannix-McNamara, 2014). Activities including, for example, raising awareness of what constitutes mental health and well-being and how to minimise the associated risks of mental ill-health are examples.

Traditionally, the primary purpose of prison was based on separation and confinement from society, punishment for crime, correction and rehabilitation to the community (Watson, Stimpson \& Hostick, 2004). However, there have been repeated calls for prisons to become more actively responsive to the health and well-being of those within their care (Santora, Espnes \& Lillefjell, 2014; WHO, 2008; WHO, 1999). Over two decades ago, the WHO established the Health in Prisons Project (HIPP) advocating the promotion of a whole-prison approach to health care, in which the combination of the health of inmates and staff in a secure environment were viewed as critical to the successful implementation of health promotion and reforming interventions (Gatherer, Moller \& Hayton, 2005). However, the Trenčín statement on prisons and mental health (WHO, 2007) continued to warn of the harms caused by prison to prisoners' mental health. The report highlighted mental health awareness 
should be central to prison health care policy (WHO, 2007). More recently, a critical review

examining health promotion practice within prisons, identified continued slow progress and a weakening of commitment, both of individual nations and the WHO, and a "worrying negative trajectory" of project support (Woodall, 2016, pg. 619).

Woodall (2016) also highlighted that in comparison to other health promoting settings, there had been a lack of prison based evaluative research that successfully demonstrated that health promoting prisons work, benefit inmates, staff or the wider community. This research gap may be partly explained by the complicating factors of restriction, control and power imbalances inherent in the prison context (Abbot, DiGiacomo, Magin \& Hu, 2018). In a qualitative study by Keogh et al., (2017), the impact of a mental health wellness workshop on prisoners was examined. The programme encouraged participants to monitor and evaluate their own mental wellness and seek appropriate support. Participants responded better to stress, adopted effective coping mechanisms, as well as feeling equipped to be a source of assistance for other prisoners. Whilst acknowledging the study limitations, it was concluded that there is strong potential in the inclusion of mental health programmes in prison.

Mental health in prison is experienced differently by males and females. Females are more likely to report clinically significant symptoms of anxiety, borderline personality features, somatic concerns and trauma-related symptoms, and are also more likely to seek and be enrolled in prison-based treatment, (Drapalski, Youman, Stuewig \& Tangney, 2013). A significant challenge to attracting males to mental health awareness programmes and their evaluation, is that male prisoners tend to adopt and project hegemonic (i.e., ruling or dominant) masculinities as self-protection (De Viggiani, 2012). This involves masking emotional vulnerabilities (Ricciardelli et al., 2015) and / or avoiding mental health related 
help-seeking behaviour for fear of reprisal, external consequences and internal costs, for example, feeling weakened or shamed for seeking assistance (Howerton et al., 2007).

Therefore, innovative ways are required to engage male prisoners in mental health awareness programmes.

Engaging and attracting prisoners to mental health awareness activities through the use of sport has been largely overlooked (Meek \& Lewis, 2012). Thus, sport may be perceived by prisoners as more acceptable context for mental health programmes than traditional didactic models. Woods, Breslin \& Hassan (2017) conducted a review of the perceived benefits of physically active sport-based interventions, ranging from 6 weeks to 9 months in duration with varying frequencies, on the psychological well-being of people in prison. The review highlighted reductions in depression, stress and anxiety, alongside increases in selfconfidence, self-esteem and pro-social identities. However, Woods \& Breslin (2019) also highlighted multiple barriers to sport-based interventions, including availability when taking place over multiple weeks and operational restrictions of the prison environment.

The complexity of co-existing factors within the prison environment, including operational restrictions and individual barriers to help-seeking, therefore requires feasible and innovative mental health awareness activities and research approaches. Within the Ministry of Justice policy paper entitled 'Preventing Suicide in England' (Department of Health, 2017), State of Mind Sport was highlighted as an innovative community based mental health and well-being initiative. The programme is aimed at raising awareness and tackling stigma, as well as encouraging individuals to seek help when needed. 
The current feasibility study therefore evaluated a pilot State of Mind Sport (SOMS)

intervention within the prison population. The aims were to (a) examine the feasibility of adopting the SOMS programme in a prison setting; and (b) test for effects of SOMS on participants' knowledge of mental health, intentions to seek help, well-being and resilience in comparison to a waiting list control group. The findings will help shape current advice available on how to use sport to integrate mental health awareness in prison settings.

\section{Method}

Participants

A total of 75 male prisoners from the same category $\mathrm{C}$ prison in England agreed to participate $(M$ age $=37.30$, S.D. $=11.01)$. The mean prison sentence length $=241.4$ months $(S . D .=$ 436.9), and mean time served in months was 38.8, (S.D. $=73.7)$. The large standard deviation values observed are due to 13 prisoners serving indeterminate life tariffs. A total of 47 prisoners $($ Mage $=38.3$, S.D. $=11.4)$ formed the intervention group, with 28 prisoners $($ Mage $=35.3, S . D .=10.2)$ participating as a control group.

Design

A mixed 2 × 2 factorial design was adopted. The between groups factor Group had two levels, intervention and control. The within groups factor Time had two levels, time-point 1 (baseline) and time-point 2 (immediately post-programme). The intervention group received a sport-based multi-component mental health awareness programme. The waiting list control group completed the baseline and post questionnaires at the beginning and end of an education class, not related to mental health and matched for SOMS intervention duration. 
The State of Mind Sport (SOMS) programme aimed to raise awareness of, and promote, psychological well-being and resilience, tackle stigma, and highlight the importance of signposting to appropriate services, with the overall aim of preventing suicide. The programme was delivered by a mental health and substance misuse nurse, and two elite level rugby players who had experienced mental health problems. There was no direct custodial experience within the delivery team and it was the first time the SOMS programme was delivered within a prison. Through collaborative working with the Suicide and Self Harm project group within the National Offender Management Service (NOMS), the programme was tailored to include a mixture of the original content (delivered to over 25,000 people in sports clubs, universities, construction and office workplaces), alongside specific contextual information relevant to the prison environment. Topics included in the programme were: (a) risks men face in relation to mental health and psychological well-being; (b) risk factors such as stigma, macho-cultures, avoidance of help-seeking behaviours and negative coping strategies; (c) markers of stress and positive coping strategies; and (d) well-being and resilience (both an understanding of the concepts and practical steps to improve). Two casestudies were presented by the former elite rugby league players who suffered from poor mental health and considered taking their own lives, prior to seeking help. Key messages they aimed to deliver included: (a) seek help/advice from someone you trust; (b) it is a strength, not a weakness to seek help; (c) respond to a mate who may be feeling down; (d) setting achievable goals and celebrating upon achievement; and (e) we are all part of a team (www.stateofmindsport.org).

\section{Procedure}

Ethical approval was granted from the Ulster University Research Ethics Committee (UREC), Office for Research Ethics Committees, Northern Ireland (ORECNI; Ref 
16/NI/0047) and the National Research Committee (NRC; Ref 2017-014). Subsequently, the prison Governor approved a pilot of the SOMS intervention and associated research to assess for evidence-based impact. To facilitate the research design, specifically the inclusion of an 8-week follow-up, all prisoners who had four months or more left to serve following conclusion of the intervention, were invited to attend the SOMS intervention. This study is inclusive of data obtained at baseline (T1) and immediately post-programme (T2) only, therefore excluding data obtained at 8-weeks follow-up (T3). SOMS flyers were designed inhouse by the prisoners and distributed to those meeting the inclusion criteria, along with a Participant Information Sheet introducing the research, explaining what it entailed, inviting questions and highlighting the voluntary nature of participating in the study.

Prisoners who volunteered to attend returned their SOMS flyers, indicating which date and session they would like to attend (two dates were offered, each with a morning and afternoon session). On attendance at the SOMS intervention, participating prisoners were provided with consent forms, given an opportunity to ask questions, and completed a suite of questionnaires detailed below under Outcome Measures, prior to and immediately after the session. To accommodate all those expressing an interest in attending the intervention, three sessions were delivered: day one PM (24 attending, 21 study participants), and day two AM (14 attending, 10 study participants), and PM (19 attending, 16 study participants). There was also a session available for staff to attend on the morning of the first day, which was not included for evaluation within the current research, which included wing staff, PE staff, healthcare and management. The intervention lasted approximately 75 minutes. The control group consisted of a convenience sample of prisoners engaging with the regime and attending their training workshops and education classes on the same days as the SOMS intervention. 
They were provided with a Participant Information Sheet and Consent Form, alongside the suite of questionnaires, with the option to participate having read both.

Focus Groups: Focus groups were held immediately following both of the sessions on day two (Group A, $n=8$; Group B, $n=7$ ). The focus group planned for day one was cancelled due to unrest in one of the prison wings and associated security concerns, with logistical and operational barriers preventing this from being re-scheduled.

\section{Outcome measures}

The Short Warwick Edinburgh Mental Well-being Scale (SWEMWBS)

The 7-item shortened version of original Warwick-Edinburgh Mental Well-being Scale (WEMWBS) was developed for assessing positive psychological well-being. It assessed psychological functioning (i.e. eudaimonic aspects of well-being: energy, clear thinking, competence, and autonomy) and feeling (cheerfulness and relaxation) and has been robustly validated (Stewart-Brown et al., 2009).

The Brief Resilience Scale

The Brief Resilience Scale (BRS) contains six items and assessed ability to bounce back or recover from stress. It is a reliable means of assessing resilience, with Cronbach's alpha ranging from $0.80-0.91$ (Smith et al., 2008).

\section{Mental Health Knowledge Schedule (MAKS)}

The Mental Health Knowledge Schedule (MAKS) comprises evidence-based knowledge in relation to stigma reduction (Evans-Lacko et al., 2010). The MAKS is based on literature review, expert consultation, and has a Cronbach's alpha $=0.65$. It comprises 6 stigma-related 
mental health knowledge areas: help-seeking, recognition, support, employment, treatment and recovery, which inquire about knowledge of mental illness conditions. A further 6 items on the MAKS assessed knowledge of specific mental illness (e.g., depression, schizophrenia and bipolar disorder) and often mistaken non-mental illnesses (grief and stress).

\section{Reported and Intended Behaviour Scale (RIBS)}

The Reported and Intended Behaviour Scale (RIBS) is a measure of mental health stigma and has demonstrated good reliability and validity, Cronbach's alpha $=0.85$ (Evans-Lacko et al., 2011). Items 1-4 address the respondent's exposure to individuals with mental health problems through examining whether they live or work with, or have a neighbour or close friend, with a mental health problem. Items 5-8 relate to intended behaviours in relation to willingness to live or work with, live nearby, or continue a relationship with someone with a mental health problem.

\section{Statistical Analysis}

Prior to performing inferential statistical analysis across time-points 1 and 2, separate between groups t-tests $(t)$ were calculated to establish if baseline differences were present between groups on any of the outcome measures. For each outcome measure, a separate 2 (Group) x 2 (Time) mixed factors Analysis of Variance (ANOVA) was calculated to determine main effects and interaction effects $(F)$. Where significant effects were observed, separate Analysis of Covariance (ANCOVA) tests were calculated to ensure these were not observed as a result of baseline scores. Prior to completing the parametric ANOVA tests, data was cleaned and checked for the following assumptions as advised by Field (2013): (a) there were no significant outliers; (b) dependent variables were normally distributed; and (c) there was homogeneity of variance. If Mauchley's test of sphericity was $<.05$, Greenhouse-Geisser 
was calculated. Statistical significance was set at $p<0.05$. Partial eta squared $\left(\eta_{p}{ }^{2}\right)$ effect size was calculated, providing an indication of what proportion of the variance in the dependent variable was attributable to the intervention. All calculations were performed using the Statistical Package for the Social Sciences (SPSS) Version 22.

\section{Focus Groups}

Fifteen prisoners volunteered to participate in two focus groups ( 8 and 7 in each group), immediately following delivery of the SOMS programme and completion of the questionnaires. A topic guide was developed exploring (a) perceived impact of participating in a sports-based mental health programme; (b) acceptability of the programme; and (c) potential challenges and improvements to the programme. A complete focus group protocol can be obtained by contacting the first author; examples questions were: 1) What were your expectations of the training course? 2) Were your expectations fulfilled? (explore reasons for or against); 3) Did the status of the course facilitators (ex-professional rugby league players) impact your experience of the training? (explore reasons for or against). Both focus groups were audio recorded and transcribed verbatim. As the feasibility study was the evaluation of a pilot programme, a General Inductive Analysis (GIA) approach was adopted to interpret focus group data. This approach was deemed appropriate as it: (a) enables researchers to condense raw textual data into a summary format; (b) facilitates the creation of linkages between research aims and summary findings from the raw data; and (c) can be used to inform a framework for interpretation of participant views (Thomas, 2006).

Trustworthiness of the data, analysis and final themes were established through a number of checks conducted throughout the study as advised by Sparkes (1998). The study included extensive participant quotes to enhance the validity of the findings, with the participant I.D. 
indicating the origin of each quote. All raw-data quotes were subjected to an audit trail that included mapping from the participant's spoken word to theme creation. Finally, detailed discussions were held between the researchers to explain and challenge emergent themes.

Results

\section{Baseline}

No significant differences were shown between the control and intervention groups at baseline for SWEMWBS, BRS, MAKS and RIBS. Mean scores and standard deviations for all measures across time-points 1 and 2 are presented in Table 1.

\begin{tabular}{|l|c|c|c|c|}
\cline { 2 - 5 } \multicolumn{1}{c|}{} & \multicolumn{2}{|c|}{ Time-point 1 (baseline) } & \multicolumn{2}{c|}{ Time-point 2 (post) } \\
\cline { 2 - 5 } \multicolumn{1}{c|}{} & Intervention & Control & Intervention & Control \\
\cline { 2 - 5 } & $\mathrm{M}(S D)$ & $\mathrm{M}(S D)$ & $\mathrm{M}(S D)$ & $\mathrm{M}(S D)$ \\
\hline SWEMWBS & $21.73(4.79)$ & $23.44(4.88)$ & $21.94(5.62)$ & 23.85 \\
& & & $4.78)$ \\
\hline MAKS & $3.07(0.96)$ & $3.35(0.88)$ & $3.11(0.90)$ & $3.38(0.89)$ \\
\hline RIBS & $21.21(3.12)$ & $20.71(2.88)$ & $23.09(2.79)$ & 20.89 \\
& $16.13(3.29)$ & $15.68(3.22)$ & $16.91(2.56)$ & $(3.06)$ \\
& & & & $(2.80)$ \\
\hline
\end{tabular}

Table 1: Outcome Measure Mean Scores and Standard Deviations

\section{Mental Health Knowledge Schedule}

The highest achievable score for the MAKS was 30 , based on the summing of responses to questions $1-6$. Table 1 shows that for the intervention group, mean score for knowledge of 
mental health at baseline $(M=21.21, S . D .=3.12)$ increased following the intervention $(M=$ 23.09 , S.D. $=2.79$ ). To compare this increase in scores with the results for the control group, a $2 \times 2$ mixed factors ANOVA was calculated. A significant main effect of group, indicating that knowledge of mental health, was greater for the intervention group, $F(1,73)=5.24, p=$ $0.03, \eta_{p}{ }^{2}=0.07$ was shown. There was also a significant interaction effect for Group and Time, $F(1,72)=4.92, p=0.03, \eta_{p}{ }^{2}=0.06$, demonstrating a significant greater improvement in mental health knowledge score from baseline to immediately post-programme for the intervention group. ANCOVA results showed that the significant intervention effect on postprogramme mental health knowledge scores remained after controlling for the baseline scores $F(1,72)=10.29, p<0.01, \eta_{p}^{2}=0.13$

Questions 7-12 of the MAKS relate to knowledge of different types of mental illness. Separate Wilcoxon $\mathrm{Z}$ tests were calculated to examine whether any change in knowledge was significant between pre and immediately-post intervention testing. Participants who received the SOMS training were more knowledgeable that stress $(Z=-3.30, p<0.01)$ was not a mental illness. There were no significant knowledge changes for grief, bipolar disorder, schizophrenia or depression for the intervention group, and no significant knowledge changes for the control group on any of the mental illnesses presented.

\section{Reported and Intended Behaviour Scale}

The highest achievable score for the RIBS was 20 , based on summing responses to questions 5-8. Higher scores were indicative of a greater willingness to engage with someone with a mental illness. For the RIBS there was no significant main effect of Group $F(1,73)=1.09, p$ $=0.30, \eta_{p}{ }^{2}=0.15$. There was a near significant within group effect, $F(1,73)=3.34, p=0.07$, $\eta_{p}{ }^{2}=0.44$, wherein both groups increased their mean scores over time (see Table 5.2), 
however this could not be attributed to the intervention as there was no significant interaction effect between Group and Time, $F(1,73)=0.47, p=0.49, \eta_{p}{ }^{2}=0.01$.

\section{Brief Resilience Scale}

For the BRS there was no significant between group, $F(1,73)=1.71, p=.20, \eta_{p}{ }^{2}=0.23$, within time, $F(1,73)=0.30, p=0.59, \eta_{p}{ }^{2}=0.004$, or interaction effect between Group and Time, $F(1,73)=0.00, p=0.985, \eta_{p}{ }^{2}=0.00$.

\section{Short Warwick Edinburgh Mental Well-being Scale}

For the SWEMWBS there was no significant main effect of Group, $F(1,73)=2.30, p=.13$, $\eta_{p}{ }^{2}=0.03$, no within group effect, $F(1,73)=2.12, p=0.66, \eta_{p}{ }^{2}=0.03$, and no significant interaction effect between Group and Time, $F(1,73)=0.19, p=0.66, \eta_{p}{ }^{2}=0.03$.

\section{Focus Group Results}

Four distinct themes emerged from the focus groups, these were Perceived Impacts, Sports Appeal, Potential Barriers to Impact and Suggested Improvements, with 12 associated subcategories (see Table 2). Quotes are presented to better illustrate each theme.

\begin{tabular}{|c|c|c|}
\hline & Theme & Categories \\
\hline \multirow{3}{*}{1} & \multirow{3}{*}{ Perceived Impacts } & a) Sense of Hope \\
\hline & & b) Sense of Perspective and Coping Efficacy \\
\hline & & c) Positive Social Networks \\
\hline \multirow{2}{*}{2} & \multirow{2}{*}{ Sports Appeal } & a) Attraction of Sport \\
\hline & & b) Sense of Legitimacy \\
\hline \multirow{4}{*}{3} & \multirow{4}{*}{ Potential Barriers to Impact } & a) Wary of Trusting Others \\
\hline & & b) Mental Health Stigma \\
\hline & & c) Lack of Appropriate Meeting Space \\
\hline & & d) Lack of Follow-up Support \\
\hline
\end{tabular}


a) Handouts

b) Prisoner Involvement

\section{Table 2: Emergent Focus Group Themes and Associated Categories}

\section{Theme: Perceived Impacts}

Sense of Hope

Participants felt they had a renewed sense of hope; that with a positive attitude, willingness to open up to others, and support from others, negative thoughts and feelings could be better dealt with. Participant B3 captured this well in the following quote:

"I think as far as this programme goes, it's good. It showed two gentlemen that have been through it and it's almost like a sign of hope. Obviously we're going through hardships now, maybe we've gone through hardships in the past and like you say, going outside is going to be a whole new world. We're going to be treated differently, we're ex-offenders now, we know it's tough getting jobs, there are going to be more hardships in the future as well. But seeing gentlemen that have been through, you couldn't imagine much worse than being disabled from the neck down, and going through that and seeing a positive attitude. I think that positivity that comes across is a big key."

\section{Sense of Perspective and Coping Efficacy}

Participants agreed that the programme offered a sense of perspective on their own personal problems. The prevailing view was that if the SOMS speakers could deal with the problems they faced (e.g., loss of identity, depression, disability, suicidal thoughts) and continue to be positive and succeed, then prisoners could also re-frame their problems more positively. As a 
result, there was also a sense of improved coping efficacy among the prisoners. Participants A4 and B4 capture these emerging themes in the following quotes:

"To hear you're paralyzed from the neck down, it's massive isn't it, and it sort of brings things into perspective. Obviously, suffering from depression anxiety and other stuff like... they've overcome massive things haven't they, and they're not dwelling on the worst, they're taking the best, if you know what I mean?"(Participant A4)

"And this is exactly how we've been feeling. None of us would be sat here if we didn't feel that... we don't want to lie about it, we do want to learn, we do want to recover... if you've got these new skills and these new tools, then we can go outside, hopefully a better person." (Participant B4)

\section{Positive Social Networks}

Reflecting on the benefit the presenters received from opening up to their friends and family, prisoners commented on the potential for benefit to be gained from confiding in their own positive social networks within prison. Similarly, there was an increased awareness of their own potential to act as a positive sounding board for prisoners at risk of poor mental wellbeing. However, this was tempered by a wariness of taking on other people's problems as they had enough to deal with; whilst others took the opposite view, that listening to other people's problems helped put theirs into a more positive perspective.

\section{Theme: Sports Appeal}

Attraction of Sport

Both the sporting context and the mental well-being theme of the session were equally attractive to participants, as voiced by Participant A1: "I think that makes it more appealing 
[the session theme], that it was ex-professional rugby players [presenting]”. However, other participants commented they had no over-riding interest in sport or awareness of the presenters. Their primary reason for attending was purely an interest in the topic: "I'd never seen him before in my life, professional rugby player and that, but because they said they'd opened up, you're thinking yes alright I can relate to that, I can listen to that... that's what you need in here, something like that."

\section{Sense of Legitimacy}

There was a connection made with the prisoners in terms of the macho culture within sports, particularly in Rugby League, and experiences within the prison of a culture which stigmatised help-seeking behaviour. Prisoners felt the life stories presented and the personal impacts described afforded them a sense of legitimacy when experiencing similar feelings of depression or anxiety for example, and that it was acceptable to open up and discuss these feelings with others:

"The reason I came was for many years I've lived this... what we call this gangster's lifestyle, where I grew up with men, so we thought we were men. But when I've been in prison now, and when your own head's on the pillow and you're on your own, nobody around, that's when you let it all out. Now I've realized I'm more at peace with myself today than what I was when I was out there thinking I was a real man. Whereas today, like that gentleman said, six foot four, 17 stone and not ashamed of crying. So I can feel like him today. That stigma, that thing. It was nice for me to come and share something. " (Participant B2)

Theme: Potential Barriers to Longer-Term Impact 
Wary of Trusting Others

Participants highlighted the crucial importance of trust and confidence within prison life and the need to ensure that other prisoners are genuinely interested in helping you. The low levels of trust made participants unwilling to open up and talk freely about these issues. Thus, within prison one had to be guarded as others will take advantage. This was particularly poignant, as having fellow prisoners to trust in was considered important in the absence of family members or friends, sources of support who were perceived as critical in positive outcomes with the SOMS presenters.

"It's hard in here because, like you're saying it's the social networks, sometimes they are easy to form, but they're formed in the wrong way so you do notice the more and more time you spend in here, you do notice the more people that are in it for themselves... it's survival, and once you experience that (and some people experience that in a hard way, I've been exploited or worse) that leads to segregation again, and trust issues. You've experienced it with one person and you start doubting everybody else even though these guys here might be honest Joes, it just might be that one person that has tainted our expectations. " (Participant B4)

"One of the big issues in prison is confidentiality. You can talk to listeners, you can talk, and all that sort of thing, then that becomes the difficulty. If somebody says, "I'm going to commit suicide, "you can't just say, "Well I'm not going to tell anybody about that," because that's a lot to take on board, because you're taking that person's life on board, but they really don't need a whisper going around the wing or the staff talking amongst themselves in an unprofessional way, as they do about things." (Participant A4)

Mental Health Stigma 


\begin{abstract}
Although the personal stories presented by the ex-elite rugby league players had presented an alternative narrative to a toxic macho culture, concerns remained of the potential for stigmatisation upon revealing a mental health concern/ issue. Participants described that these concerns regarding stigmatisation were applicable both within prison and on release, and could have a negative impact on how you were perceived by the prison regime, for example, in relation to parole.
\end{abstract}

\title{
Lack of Appropriate Meeting Space
}

A lack of appropriate space for people to informally meet and discuss their issues or concerns in confidence was identified as an environmental barrier within the prison.

"People find it hard to talk just one on one. If you are in a group basis, you find it easy ... helps people in a group to talk. The end of every landing here, you have a room with nothing in it, couple of chairs... why can't that room be opened for people who's going to sit in there? (Participant A2); “Unfortunately, it's down to operational services to get say a group of us, us men, into a room... you'd have to have an officer watching" (Participant A3).

Lack of Follow-up Support

Finally, questions were raised regarding what follow-up services might be provided within the prison in terms of support for well-being specifically in connection with the information presented.

“You talk to us today, and you're not here tomorrow, is anything going to happen to us in here tomorrow to help us? Is there anything going to happen [as a result] of us talking today now?" (Participant A3)

\section{Theme: Suggested Improvements}




\section{Mental Health Information Resources}

The first suggestion was the provision of related materials, both as paper handouts for those attending and those who were not ready to openly attend a programme related to mental health awareness. Participants recommended that information handouts capturing the key learning points and tools discussed would serve as a useful aid. Participant B6 commented " $I$ took notes as fast as I could, but if you had a handout that would be better." Another participant highlighted the potential benefit of associated materials for those not attending:

"You know some people had the courage to come today, but like we had talked about before, we know there's a stigma and there's people afraid to come forward. Some people need that kind of anonymity, either somebody they can trust or picking up a leaflet. So if there was information that could be supplied... either to get in contact with yourselves about the programmes that they run or the information about where we can find the relevant information if we needed it." (Participant A1)

\section{Prisoner Involvement}

The second suggestion for improvement was that alongside the stories of the ex-sportsmen, similar sessions could be run with current or ex-offenders who had experiences of successfully coping with mental health issues, as this would be very relatable to the prisoners: "I think one of the things that needs to be done is people who experience the situation with mental health problems and see what situation they've been in in prison, their problems, how they've been able to get help or not get help. Whether it's been appropriate, that sort of thing. I think you need a case study of people willing to do that." (Participant A6) 


\section{Discussion}

The aims of the current study were to: (a) examine the feasibility of adopting the SOMS programme in a prison setting; and (b) test for effects of SOMS on participants' knowledge of mental health, intentions to seek help, well-being and resilience in comparison to a waiting list control group.

Prisoners in the intervention group increased knowledge of mental health and ability to correctly classify types of mental illness compared to those in a control group. These findings demonstrate that a short mental health awareness programme using sport can successfully elevate knowledge of mental health issues within the prison population. In contrast to the increase in knowledge of mental health, there was no associated significant increase in intentions to engage and offer support to those with mental health concerns. However, focus group results showed prisoners intentions to more readily act as a sounding board for others following the SOMS programme, alongside an increased willingness to open up to others, reflecting similar findings from a mental health wellness programme (Keogh et al, 2017).

We offer two explanation for the variance in findings across the two research methods. The first being that positive prisoner intentions to help others expressed within the focus groups, were tempered by the concern of other prisoners that they had enough to deal with themselves before acting as a confidant for fellow prisoners, which may have impacted responses to the items on the RIBS. The second issue may be the suitability of RIBS as a measure within the prison setting. Items enquire about the respondent's intentions to: "live with", "work with", "work nearby" and "continue a relationship" with someone suffering from a mental illness. Within prison, there is minimum autonomy in relation to these (and many other) variables, (De Viggiani, 2012). Also, the point at which prisoners might be able 
to make such choices upon release can be many years in the future and therefore may be viewed as abstract and hard to fully imagine. These two factors may have combined to impact the validity of the RIBS questionnaire in its current format, as despite reporting robust psychometric properties they are not widely tested in prisoner populations. Future studies should therefore consider an altered version of the RIBS, presenting scenarios better reflecting probable personal interactions within the prison environment. Similarly, if adequate participant numbers were recruited specific reliability and validity analyses could be completed.

No significant differences were recorded between groups for prisoner scores on psychological well-being and resilience immediately post-programme. Psychological wellbeing, as measured by SWEMWBS, reflects self-perceptions of positive 'functioning' more than 'feeling' (Tenant et al., 2007). Therefore, the absence of significantly enhanced levels of psychological functioning, which relate to personal feelings of value and meaning in life (Huta, 2016), is perhaps not surprising immediately following a 75-minute programme. Building resilience is an interactive evolving process (Herrman et al., 2011), which develops when provided with new adaptive coping strategies. Therefore, the messages and coping strategies delivered within SOMS, targeting increased well-being and resilience, would require increased time to become embedded and potentially reflected in the outcome measures used.

An emergent theme from the focus groups was prisoners' increased willingness to confide in fellow inmates and discuss their vulnerabilities as a positive coping mechanism. This is in contrast to the stereotypical male response of stoicism when faced with emotional distress within prisons (Ricciardelli et al., 2015). However, the translation of any increase in 
intentions to discuss mental health concerns into actions had to compete with an opposing theme, i.e., the perceived likelihood of being stigmatised for doing so. Fellow prisoners and the prison system were both identified as sources of stigmatisation. This view persisted despite the programme addressing issues regarding mental health stigma, and could act as a barrier to improved mental well-being. Lack of trust in other prisoners, prison staff, and the potential for admissions of low mental well-being to negatively impact parole hearings, were perceived as barriers to adopting a more transparent approach to discussing well-being.

Fear of stigmatisation, lack of trust in other prisoners and the prison system all replicate findings from previous research examining help-seeking behaviour within prisons (De Viggiani, 2012; Howerton et al., 2007; Ricciardelli et al., 2015; Skogstad, Deane \& Spicer, 2006). Avoidance of help-seeking behaviours is also linked to the maintenance of prison masculinities and a weighing up of the risks associated with such disclosures (Ricciardelli et al., 2015). Considering feasibility, future programmes that are designed as community-based programmes then transferred into a prison, must therefore ensure they gain a deep understanding of the nuances and specific challenges prisoners face when attempting to adopt coping strategies presented to them. Failure to recognise and cater for these contextual difficulties may open the potential for negative impacts rather than benefits within prison. Results from this study show that providing safe spaces for developing trusted relationships with other prisoners, providing on-going support following workshops to develop and implement new coping skills, and the inclusion of prisoner testimonies could help mitigate these risks, increase coping-efficacy and overall programme feasibility.

An emergent theme from the focus groups was that SOMS, as a mental health awareness programme with its origins in the hyper masculine sport of rugby league, was effective in at 
least triggering a small shift in the restrictive toxic masculinities present within the prison environment (Kupers, 2005). The use of sport as a catalyst, specifically ex-professional rugby league players, was perceived to afford a legitimacy to the vulnerabilities of the prisoners and offer an alternative empowering narrative to that usually encountered prior to and within prison. This is an important finding regarding programme feasibility, as although several focus group participants reported motivation to attend due to the sport-based nature of the programme, many others had no over-riding interest in sport and were attending due to the focus on mental health. Importantly, those with no sporting interest still felt the messages delivered by the ex-professional rugby players resonated with their current situation.

Combined with an increased willingness to seek help from others and sense of perspective on their own problems, prisoners reported an increased sense of hope immediately following the SOMS programme. Prisoners referred to increased hoped they could overcome personal difficulties and transition through the gate a better person. This is an important outcome as a sense of hopelessness has been consistently linked with self-harm and suicide (Fazel, Cartwright, Norman-Nott \& Hawton, 2008; Gooding et al., 2017; Konrad et al., 2007). We speculate that SOMS, as a result of increasing prisoner hope, will also impact coping efficacy, therefore having significant positive improvements in mental well-being within prisons.

There were several limitations within the current research, partly reflecting the methodological landmines inherent in prison research (Schlosser, 2008). Considering sample size, although 75 prisoners were successfully recruited, this prohibited stratified analysis across variables such as offence committed or sentence length, which may have revealed important differences in outcome measures across certain offences. Secondly, although the 
inclusion of a control group was a strength, there was no randomisation of participants to groups due to prison restrictions, introducing the potential for bias. The brief nature of the intervention and limited time between questionnaire administration will have restricted the possibility of change in some outcome measures, such as resilience. Focus group results immediately following the intervention revealed important findings, for example, an increased sense of hope and greater help-seeking intentions, however there was no measure of whether associated behavioral changes persisted and translated into positive actions over time.

The outcomes of the research and limitations discussed give rise to several recommendations for future research. Future studies should include additional measures to validate some of the findings discussed, for example, those related to increased help-seeking intentions and decreased feelings of hopelessness. Programme development, and associated evaluations, could also consider the inclusion of behaviour change theories, such as the Theory of Planned Behaviour (Ajzen, 1985), alongside side Self-Determination Theory (Deci \& Ryan, 2000) in relation to help-seeking behaviours. A longitudinal assessment would offer insights into the longer-term feasibility and impacts, if any, of participating in the programme, particularly in coping with ongoing incarceration or in negotiating the transition from custody to community. Likewise, to increase the feasibility of future programmes originating within a community setting, a focus is required on how to embed and maxmise follow-up support and guidance for prisoners to maintain any positive affective, behavioural or cognitive changes that may have emerged as a result of the programme. Consideration should also be given as to whether programme duration or frequency could be increased to maximise impact.

\section{Conclusion}


A short sport-based mental health awareness intervention was well received by a cohort of male prisoners who, as a result, increased their knowledge of mental health issues.

Participants reported increased intentions to adopt more positive coping strategies, to seekhelp and engage with others. To further increase programme feasibility several suggestions for programme enhancements were made. These include the development of supporting programme resources, the inclusion of prisoner case-studies and the provision of appropriate and informal safe meeting spaces for prisoners. Given the current levels of mental illness, suicide and self-harm within prisons (National Audit Office, 2017; Wildeman \& Wang, 2017), and lack of prison based evaluative studies to provide reliable evidence of what works (Woodall, 2016), this study makes an important contribution using a mixed methods approach to the health promoting prison agenda. 


\section{References}

Abbott, P., DiGiacomo, M., Magin, P., \& Hu, W. (2018). A scoping review of qualitative research methods used with people in prison. International Journal of Qualitative Methods, 17, 1-15.

Ajzen, I. (1985). From intentions to actions: A theory of planned behavior. In Action control (pp. 11-39). Springer, Berlin, Heidelberg.

Department of Health. (2017). Preventing suicide in England: Third progress report of the cross-government outcomes strategy to save lives. Retrieved from:

https://assets.publishing.service.gov.uk/government/uploads/system/uploads/attachment_data /file/582117/Suicide_report_2016_A.pdf

Deci, E. L., \& Ryan, R. M. (2000). The" what" and" why" of goal pursuits: Human needs and the self-determination of behavior. Psychological inquiry, 11(4), 227-268.

De Viggiani, N. (2012). Trying to be something you are not: Masculine performances within a prison setting. Men and Masculinities, 15(3), 271-291.

Drapalski, A. L., Youman, K., Stuewig, J., \& Tangney, J. (2009). Gender differences in jail inmates' symptoms of mental illness, treatment history and treatment seeking. Criminal Behaviour and Mental Health, 19(3), 193-206.

Evans-Lacko, S., Little, K., Meltzer, H., Rose, D., Rhydderch, D., Henderson, C., \& Thornicroft, G. (2010). Development and psychometric properties of the mental health knowledge schedule. The Canadian Journal of Psychiatry, 55(7), 440-448.

Evans-Lacko, S., Rose, D., Little, K., Flach, C., Rhydderch, D., Henderson, C., \& Thornicroft, G. (2011). Development and psychometric properties of the reported and intended behaviour scale (RIBS): a stigma-related behaviour measure. Epidemiology and Psychiatric Sciences, 20(3), 263-271. 
Fazel, S., Hayes, A. J., Bartellas, K., Clerici, M., \& Trestman, R. (2016). Mental health of prisoners: prevalence, adverse outcomes, and interventions. The Lancet Psychiatry, 3(9), 871881.

Field, A. (2013). Discovering statistics using IBM SPSS statistics. Sage.

Gatherer, A., Moller, L., \& Hayton, P. (2005). The World Health Organisation European health in prison project after 10 years: persistant barriers and achievements". American Journal of Public Health, 95(10), 1696-700

Gooding, P. A., Tarrier, N., Dunn, G., Awenat, Y., Shaw, J., Ulph, F., \& Pratt, D. (2017). Psychological characteristics and predictors of suicide probability in high-risk prisoners. Criminal Justice and Behavior, 44(3), 321-335.

Herrman, H., Stewart, D. E., Diaz-Granados, N., Berger, E. L., Jackson, B., \& Yuen, T. (2011). What is resilience? The Canadian Journal of Psychiatry, 56(5), 258-265.

Howerton, A., Byng, R., Campbell, J., Hess, D., Owens, C., \& Aitken, P. (2007). Understanding help seeking behaviour among male offenders: qualitative interview study. BMJ, 334(7588), 303.

Huta, V. (2016). Eudaimonic and hedonic orientations: Theoretical considerations and research findings. In Handbook of Eudaimonic Well-Being (pp. 215-231). Springer International Publishing.

Keogh, B., McBennett, P., deVries, J., Higgans, A., O’Shea, M., \& Doyle, L. (2017). Prisoners perceptions of a mental health wellness workshop. International Journal of Prisoner Health, 13(2), 81-90.

Konrad, N., Daigle, M. S., Daniel, A. E., Dear, G. E., Frottier, P., Hayes, L. M., ... \& Sarchiapone, M. (2007). Preventing suicide in prisons, Part I: Recommendations from the International Association for Suicide Prevention Task Force on Suicide in Prisons. Crisis: The Journal of Crisis Intervention and Suicide Prevention, 28(3), 113. 
Kupers, T. A. (2005). Toxic masculinity as a barrier to mental health treatment in prison. Journal of clinical psychology, 61(6), 713-724.

MacNamara, C., \& Mannix-McNamara, P. (2014). Placing the promotion of health and wellbeing on the Irish prison agenda - the complexity of health promotion in Irish prisons. Irish Journal of Applied Social Studies, 14(1), 49-59.

Meek, R., \& Lewis, G. (2012). The role of sport in promoting prisoner health. International journal of prisoner health, 8(3/4), 117-130.

National Audit Office (2017). Mental health in prisons. Retrieved from:

https://www.nao.org.uk/wp-content/uploads/2017/06/Mental-health-in-prisons-Summary.pdf

Ricciardelli, R., Maier, K., \& Hannah-Moffat, K. (2015). Strategic masculinities:

Vulnerabilities, risk and the production of prison masculinities. Theoretical

Criminology, 19(4), 491-513.

Ryan, R. M., \& Deci, E. L. (2001). On happiness and human potentials: A review of research on hedonic and eudaimonic well-being. Annual Review of Psychology, 52(1), 141-166.

Santora, L., Arild Espnes, G., \& Lillefjell, M. (2014). Health promotion and prison settings. International Journal of Prisoner Health, 10(1), 27-37.

Schlosser, J. A. (2008). Issues in interviewing inmates: Navigating the methodological landmines of prison research. Qualitative Inquiry, 14(8), 1500-1525.

Skogstad, P., Deane, F. P., \& Spicer, J. (2006). Social-cognitive determinants of help-seeking for mental health problems among prison inmates. Criminal Behaviour and Mental Health, 16(1), 43-59.

Smith, B. W., Dalen, J., Wiggins, K., Tooley, E., Christopher, P., \& Bernard, J. (2008). The brief resilience scale: assessing the ability to bounce back. International journal of behavioral medicine, 15(3), 194-200. 
Sparkes, A. (1998). Validity in qualitative inquiry and problems of criteria: Implications for sport psychology. The Sport Psychologist, 12, 363-386.

Stewart-Brown, S., Tennant, A., Tennant, R., Platt, S., Parkison, J., \& Weich, S. (2009)

Internal Construct Validity of the Warwick-Edinburgh Mental Well-being Scale

(WEMWBS): A Rasch analysis using data from the Scottish Health Education Population

Survey. Health and Quality of Life Outcomes, 9, 7-15

Thomas, D. R. (2006). A general inductive approach for analyzing qualitative evaluation data. American Journal of Evaluation, 27(2), 237-246.

Watson, R., Stimpson, A., \& Hostick, T. (2004). Prison health care: a review of the literature. International Journal of Nursing Studies, 41(2), 119-128.

World Health Organization. (1999) Mental Health Promotion in Prison: Report on a WHO Meeting. World Health Organisation, Regional Office for Europe, Copenhagen.

World Health Organization. (2014a). Mental health: A state of well-being. Geneva, Switzerland: World Health Organization.

World Health Organization. (2007), Health in prisons: A WHO guide to the essential in prison health, World Health Organisation, Copenhagen.

World Health Organization. (2008), Trenčin statement on prisons and mental health. Copenhagen: WHO Regional Office for Europe.

World Health Organization. (2014), Prisons and health. Retrieved from http://www.euro.who.int/_data/assets/pdf_file/0005/249188/Prisons-and-Health.pdf

Wildeman, C., \& Wang, E. A. (2017). Mass incarceration, public health, and widening inequality in the USA. The Lancet, 389(10077), 1464-1474.

Woodall, J. (2016). A critical examination of the health promoting prison two decades on. Critical Public Health, 26(5), 615-621. 


\author{
Woods, D., Breslin, G., \& Hassan, D. (2017). A systematic review of the impact of SBIs on \\ the psychological well-being of people in prison. Mental Health and Physical Activity 12, 50- \\ 61.
}

Woods, D. \& Breslin, G. (2019). Reflections on Implementing the Active Choices Rugby Programme to enhance mental health and wee-being of young men in prison. In G. Breslin \& G. Leavey (Eds.), Mental Health and Well-Being Interventions in Sport (pp. 128-143). Abingdon, Oxon: Routledge. 
1

7

8

Table 1: Outcome Measure Mean Scores and Standard Deviations

\begin{tabular}{|l|c|c|c|c|}
\cline { 2 - 5 } \multicolumn{1}{c|}{} & \multicolumn{2}{c|}{ Time-point 1 (baseline) } & \multicolumn{2}{c|}{ Time-point 2 (post) } \\
\cline { 2 - 5 } & Intervention & Control & Intervention & Control \\
\cline { 2 - 5 } & $\mathrm{M}(S D)$ & $\mathrm{M}(S D)$ & $\mathrm{M}(S D)$ & $\mathrm{M}(S D)$ \\
\hline SWEMWBS & $21.73(4.79)$ & $23.44(4.88)$ & $21.94(5.62)$ & 23.85 \\
\hline \multirow{2}{*}{ MAKS } & $3.07(0.96)$ & $3.35(0.88)$ & $3.11(0.90)$ & $3.38(0.89)$ \\
\hline \multirow{2}{*}{ RIBS } & $21.21(3.12)$ & $20.71(2.88)$ & $23.09(2.79)$ & 20.89 \\
& & & & $(3.06)$ \\
\hline
\end{tabular}


Table 2: Emergent Focus Group Themes and Associated Categories

\begin{tabular}{|c|c|c|}
\hline & Theme & Categories \\
\hline \multirow{3}{*}{1} & \multirow{3}{*}{ Perceived Impacts } & a) Sense of Hope \\
\hline & & b) Sense of Perspective and Coping Efficacy \\
\hline & & c) Positive Social Networks \\
\hline \multirow{2}{*}{2} & \multirow{2}{*}{ Sports Appeal } & a) Attraction of Sport \\
\hline & & b) Sense of Legitimacy \\
\hline \multirow{4}{*}{3} & \multirow{4}{*}{ Potential Barriers to Impact } & a) Wary of Trusting Others \\
\hline & & b) Mental Health Stigma \\
\hline & & c) Lack of Appropriate Meeting Space \\
\hline & & d) Lack of Follow-up Support \\
\hline \multirow{2}{*}{4} & \multirow{2}{*}{ Suggested Improvements } & a) Handouts \\
\hline & & b) Prisc \\
\hline
\end{tabular}

